\title{
A P(V)-Platform for Oligonucleotide Synthesis
}

Authors: Yazhong Huang, ${ }^{3 \dagger}$ Kyle W. Knouse, ${ }^{1 \dagger}$ Shenjie Qiu, ${ }^{2 \dagger}$ Wei Hao, ${ }^{1+}$ Natalia M. Padial, ${ }^{1 *}$ Julien C. Vantourout, ${ }^{1 *}$ Bin Zheng, ${ }^{2}$ Stephen E. Mercer, ${ }^{3}$ Javier O. Lopez, ${ }^{1}$ Rohan Narayan, ${ }^{1}$ Richard E. Olson, ${ }^{3}$ Donna G. Blackmond, ${ }^{1}$ Martin D. Eastgate, ${ }^{2 *}$ Michael A. Schmidt, ${ }^{2 *}$ Ivar M. McDonald,,$^{3 *} \&$ Phil S. Baran ${ }^{1 *}$

\author{
Affiliations: \\ ${ }^{1}$ Department of Chemistry, The Scripps Research Institute, 10550 N. Torrey Pines Rd., La Jolla, \\ CA 92037. \\ ${ }^{2}$ Chemical Process Development, Bristol Myers Squibb, One Squibb Dr., New Brunswick, NJ \\ 08903. \\ ${ }^{3}$ Small Molecule Drug Discovery, Bristol Myers Squibb, 100 Binney St., Cambridge, MA 02142. \\ *Correspondence to: martin.eastgate@bms.com, michael.schmidt@bms.com, \\ ivar.mcdonald@bms.com, and pbaran@scripps.edu \\ $\dagger$ These authors contributed equally to this work. \\ $\$$ These authors contributed equally to this work.
}

\begin{abstract}
:
The early promise of gene-based therapies is currently being realized at an accelerated pace with over 155 active clinical trials for antisense compounds and multiple FDA-approved oligonucleotide therapeutics. Fundamental advances in this area are vital and present an unprecedented opportunity to both address disease states that have been resistant to other common modalities and improve the significant sustainability challenges associated with production of these complex molecules on a commercial scale. The advent of phosphoramidite coupling chemistry and solid-phase synthesis 40 years ago democratized oligonucleotide synthesis to the scientific community, paving the way for many of these stunning developments. The reliability and generality of this approach for the preparation of native phosphate-diesters is attributed to the high reactivity of phosphorus when in the $\mathrm{P}(\mathrm{III})$-oxidation state versus the desired $\mathrm{P}(\mathrm{V})$, as it enables rapid P-heteroatom bond formation. However, the growing demand for more diverse phosphorus-based linkages has challenged the limits of this technology. For example, the phosphorothioate (PS) linkage, which stabilizes oligonucleotides towards nuclease cleavage, is universally employed in current oligonucleotide therapeutics but is generally incorporated in racemic form. Stereodefined PS oligonucleotides may have desirable biological and physical properties but are accessed with difficulty using phosphoramidite chemistry. Here we report a flexible and efficient $[\mathrm{P}(\mathrm{V})]$-based platform that can install a wide variety of phosphate linkages at will into oligonucleotides. This approach uses readily accessible reagents and can efficiently install not only stereodefined or racemic thiophosphates, but can install any combination of ( $S, R$ or $r a c)$-PS with native phosphodiester $\left(\mathrm{PO}_{2}\right)$ and phosphorodithioate $\left(\mathrm{PS}_{2}\right)$ linkages into DNA and other modified nucleotides. Importantly this platform easily accesses this diversity under a standardized coupling protocol with sustainably prepared, stable, $\mathrm{P}(\mathrm{V})$ reagents.
\end{abstract}


In traditional small molecule drug discovery, the features that determine target specificity and pharmacokinetics [DMPK (distribution, metabolism, pharmacokinetics)] are usually inextricably linked. ${ }^{1}$ Oligonucleotide-based therapeutics, on the other hand, have been referred to as "informational-drugs" wherein the pharmacophore and PK properties can, in theory, be separately optimized since the nucleoside sequence directly determines the former while the unifying chemistry (the phosphate linkages used to couple the nucleosides) largely affects the latter. ${ }^{2}$ Advances in organic synthesis have had a profound impact on the ability of modern medicinal chemistry to target and rapidly access increasingly complex small molecule leads. In contrast, as the range of oligonucleotide sequences and conceivable phosphate linkages has expanded, the fundamental chemistry used to enable their synthesis has largely remained unchanged despite numerous refinements and improvements. The incorporation of varied phosphate linkages has been documented to have a profound impact on both the properties and efficacy of the resulting structures. $^{3}$ The hypothetical chimeric sequence (1) illustrated in Figure 1, adorned with four different phosphorus-based linkages and multiple sugar backbones, tests the limits of the scope of existing methods. There are no published examples of sequences bearing this combination of chemical modifications. However, it can be anticipated that accessing new dimensions in structural space will be critical to the continued evolution of antisense therapeutics. The impact of judiciously designing backbone chemical modifications in therapeutic oligonucleotides is only beginning to be realized. Recent examples from Wave Life Sciences Ltd. have shown that incorporation of select $\mathrm{PO}_{2}$ linkages into a stereodefined $\mathrm{P}(\mathrm{S})$ backbone generated more potent and selective oligonucleotides when compared to previously published candidates. ${ }^{4,5}$ Similarly, work by Roche and Silence Therapeutics have shown that judicious incorporation of $\mathrm{PS}_{2}$ linkages into a stereorandom $\mathrm{P}(\mathrm{S})$ backbone generates more potent and stable leads. ${ }^{6,7}$ The opportunity to install broader combinations and variations, in any order, at will thus presents a new step in the evolution of oligonucleotide therapeutics and requires the invention of enabling science, one such step is described herein. Looking to the future, this newly developed reagent suite has a dramatically improved potential for manufacturing applications due to reduced step counts and reduced waste stream associated with $\mathrm{P}(\mathrm{V})$ vs. $\mathrm{P}(\mathrm{III})$ (see SM for further discussion).

Additionally, the commercialization of oligonucleotides and, more specifically, phosphorothioate antisense oligonucleotides (PS-ASOs) faces significant challenges. ${ }^{8}$ In the latter case, traditional methods make the key phosphate bonds unselectively, resulting in products being a mixture of diastereomers (up to 131,072 isomers for a standard 18-mer ASO), complicating product purification and the assessment of product quality. ${ }^{9,}{ }^{10}$ In general, the P(III) method of synthesis also requires vast amounts of reagents and solvents, ${ }^{11}$ both during oligo synthesis itself and during monomer formation, significantly impacting the sustainability for commercial manufacture - an aspect that is further complicated by the instability of the $\mathrm{P}(\mathrm{III})$ reagents themselves (which can be sensitive to both air and moisture). Methods for the asymmetric synthesis of ASOs suffer from even greater levels of environmental impact. ${ }^{12,13}$ A more stable, controlled, and stereoselective reagent platform may allow for improved synthetic approaches, such as synthesis via blockmers (short dimers or trimers made through a standard small molecule methods, prior to fragment coupling on solid phase - significantly improving efficiency and product quality). ${ }^{14}$ The ability to easily make single isomer species from stable species/fragments will aid our control over product quality, and has the potential to enable commercialization of these novel therapeutics 
sustainably (i.e. through enhanced regulatory control of these complex molecules). Thus, improved methods of oligonucleotide synthesis, amenable to standard methods of automation, would have a near-immediate translational impact, enabling both the interrogation of greater linker permutations in drug discovery and better, more sustainable commercialization [see SI for process mass intensity (PMI) analysis]. ${ }^{3}$

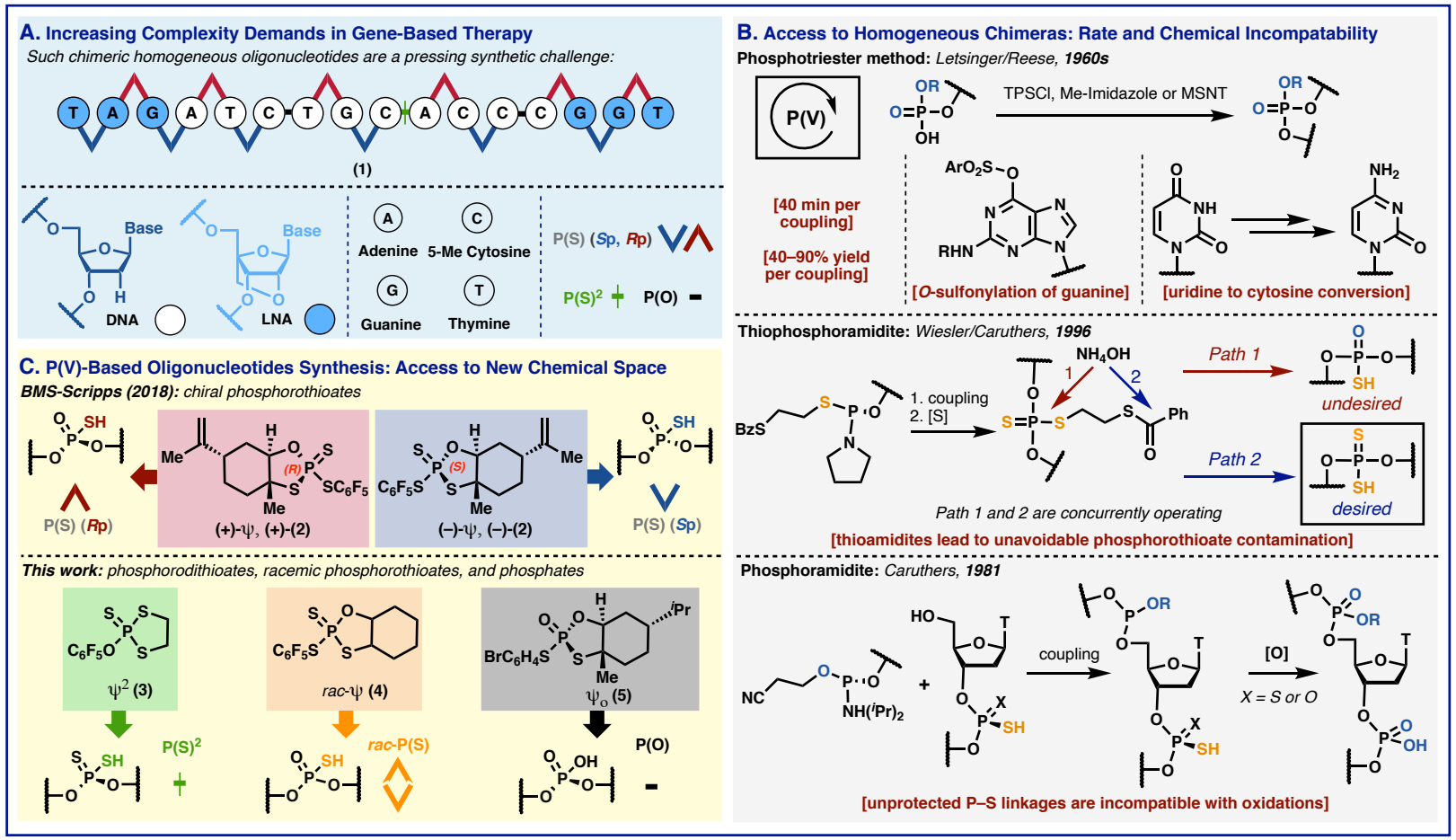

Fig. 1. Introduction and background (A) Inspiration, (B) Challenges facing $P(V)$ based synthesis (C) Recent developments and this work. i-Pr, iso-propyl; Me, methyl; Bz, benzoyl; [O], oxidation; [S], sulfurization

We recently demonstrated a practical approach to address two of these linkage types (mainly in the context of DNA), stereopure phosphorothioates (R-PS and $S$-PS), with the disclosure of the phosphorus sulfur incorporation (dubbed PSI or $\psi$ ) reagents. However, this initial study did not address the installation of two important achiral linkages: phophorodithioates and native phosphate diesters. ${ }^{15}$ Additionally, although a proof of concept for the application of $\psi$ using a commercial oligonucleotide synthesizer was presented, the enabling protocol was not optimized for general use. Three main challenges therefore needed to be overcome to access chimeric products such as (1) regardless of the approach employed (Figure 1B); 1) the rate and chemoselectivity of P(V) vs $\mathrm{P}(\mathrm{III})$ couplings must be established; 2) new reagents to access $\mathrm{PS}_{2}$ and $\mathrm{PO}_{2}$ linkages must be invented, ones that can selectively form the desired products under identical coupling conditions to PSI; and 3) the conditions for P(V) Solid-Phase Oligonucleotide Synthesis (SPOS) must be established that are mild, allowing compatibility in the formation of phosphorodithioate linkages, alongside nominal phosphodiesters, in one oligonucleotide construct.

Addressing these challenges was nontrivial — with significant lore and reactivity concerns to address. ${ }^{16-20}$ For example, the rate associated with $\mathrm{P}(\mathrm{V})$-based reagents has historically been 
viewed as too sluggish to ever compete with the P(III)-manifold. ${ }^{21-23}$ Such approaches also had chemoselectivity challenges in that the guanine and thymidine bases would interfere in sequential couplings, reacting with the coupling reagents. ${ }^{24}$ Employing P(III)-based reagents to install phosphorodithioate $\left(\mathrm{PS}_{2}\right)$ linkages is less than ideal. For instance, Caruthers reported a protocol based on protected thiophosphoramidites that requires discreet oxidation and deprotection steps. ${ }^{25}$ Unfortunately, one invariably co-isolates a nearly inseparable phosphorothioate byproduct in ca. $5-10 \%$ yield depending on the sequence and deprotection conditions employed, thus placing a cloud of uncertainty around the level of $\mathrm{PS}_{2}$ incorporation obtained, and the distribution of PSO isomer contamination, with its implementation. Despite this serious issue, this method is still commonly used as there are simply no viable alternatives. ${ }^{26}$ Finally, chimeric sequences with multiple types of linkages such as phosphodiesters $\left(\mathrm{PO}_{2}\right)$ and $\mathrm{PS}_{2}$ could be desired by medicinal chemists, but only certain combinations (PS/PO, PS/PS $\left.2, \mathrm{PO} / \mathrm{PS}_{2}\right)$ are present in the literature. Even a hybrid synthetic approach that merges P(III)-based phosphoramidite chemistry with $\mathrm{P}(\mathrm{V})$ reagents is unworkable as it suffers from a lack of chemoselectivity (vide infra) as oxidation of $\mathrm{P}(\mathrm{III})$ to $\mathrm{P}(\mathrm{V})$ requires a protecting group on exposed $\mathrm{PS} / \mathrm{PS}_{2}$ linkages to avoid desulfurization.

In this disclosure, three new reagent systems are described $\left[\psi^{2}(3), r a c-\psi(4)\right.$, and $\left.\psi^{\mathrm{O}}(5)\right]$ that, when combined with the previously reported $[(+)-\psi,(+)-2)]$ and $[(-)-\psi,(-)-2]$, provide a unified $\mathrm{P}(\mathrm{V})$-approach that entirely departs from the rubric of $\mathrm{P}(\mathrm{III})$-based oligonucleotide synthesis and enables the at will and controlled synthesis of specific chimeric oligonucleotides (Figure 1C). Along with these new reagents are protocols for their unified application in commercial synthesizers using a single coupling protocol which spans all coupling types. This redox-neutral platform, based on the native $\mathrm{P}(\mathrm{V})$-oxidation state, challenges past assumptions of sluggish reactivity and enables access to five relevant P-linkages across a range of sugar backbones (DNA and LNA) and bases (A, T, G, mC) in one oligonucleotide construct. Aside from enabling straightforward access to a wide range of chimeric oligonucleotides, the implementation of this new protocol benefits from a reduced reliance on protecting group chemistry (which eliminates the labile cyanoethyl group and therefore acrylonitrile production upon deprotection), ${ }^{27,}{ }^{28}$ bespoke additives, ${ }^{29}$ and redox fluctuations. It is also of note that this new $\mathrm{P}(\mathrm{V})$ platform eliminates one full step in the standard SPOS protocol (namely the phosphorous oxidation).

Figure 2 outlines the synthesis of $\psi^{2}$ and $\psi^{\mathrm{O}}$, reagents for the incorporation of phosphorodithioate and native phosphodiester linkages, respectively. The development of these reagents required extensive experimentation, whereas the synthesis of $r a c-\psi$ was relatively trivial and proceeded by analogy to $\psi$ using cyclohexene oxide (see SI). Fully sulfurized versions of diphosphate esters, known as phosphorodithioate linkages, are isopolar and isostructural analogs of phosphates that are completely stable to nucleases, ${ }^{30}$ while maintaining the ability to form stable duplexes and elicit desirable mRNA cleavage by RNAse-H without the complexity of chirality at phosphorus. ${ }^{31}$, 32 The pioneering work of Stec on phospholane heterocycles, which inspired the development of $(+)-\psi$ and $(-)-\psi$, was an essential precedent for the present work. ${ }^{19}$ In 1995 it was disclosed that dithiaphospholanes could be installed onto nucleosides and coupled to afford dinucleotides incorporating a phosphorodithioate linkage albeit requiring a separate oxidation step to install sulfur, and a toxic and unstable (explosive) reagent (Figure 2A).). ${ }^{18}$ Our P(V)-centric study thus built on the lessons of these two precedents with the goals of eliminating the extraneous oxidation 
step and dangerous reagents. Upon identification of the optimal leaving group (21 evaluated, see SI for Hammett correlations of leaving groups) and ring size (two evaluated), inexpensive $\mathrm{P}_{2} \mathrm{~S}_{5}$ could be combined with pentafluorophenol followed by capping of $\mathrm{P}(\mathrm{V})$ intermediate (6) with thiirane to generate $\psi^{2}$ on large scale $(>100 \mathrm{~g})$. In this way, unsafe P(III)-chemistry was avoided, and a stable and viable $\mathrm{P}(\mathrm{V})$ reagent was developed.

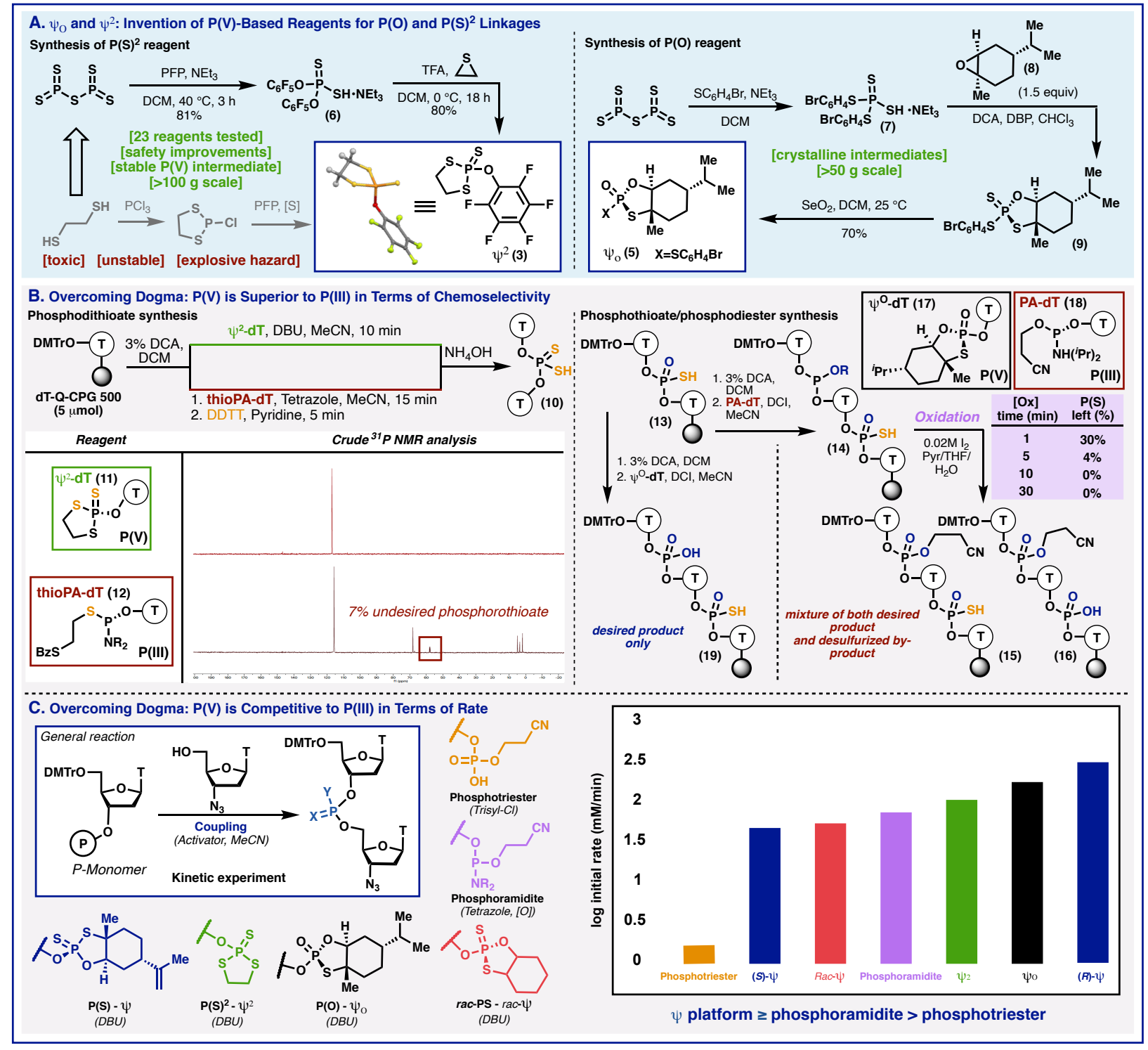

Fig. 2. Development of a fully $P(V)$ platform for oligonucleotide synthesis (A) Reagent development, (B) Overcoming chemoselectivity challenges (C) Overcoming historical P(V) rate challenges. PFP, pentafluorophenol; TFA, trifluoroacetic acid; DCA, dichloroacetic acid; DBP, dibutylphosphate; [S], sulfurization; DDTT, 3-((Dimethylamino-methylidene)amino)-3H-1,2,4dithiazole-3-thione; DCI, 4,5-dicyanoimidazole; DBU, 1,8-Diazabicyclo[5.4.0]undec-7-ene.

Oligonucleotides with native phosphodiester linkages have poor pharmacokinetics and are rapidly degraded by nucleases, but their use is invaluable in routine molecular biology and diagnostic 
settings. ${ }^{33}$ In addition, limited PO linkages are incorporated into current antisense oligonucleotides. To the best of our knowledge, there are no known $\mathrm{P}(\mathrm{V})$-based reagents for achieving P(III)-competitive reactivity and chemoselectivity that are stable and amenable to automated synthesis. ${ }^{34}$ Although the design of such a reagent benefited from the lessons of our previous studies, it was repeatedly thwarted by the challenge of identifying both a highly reactive and stable entity upon loading to a monomer. We evaluated nearly 30 different backbones along with three different leaving groups before arriving at $\psi^{\mathrm{O}}$ (see SI for comprehensive summary). The backbone optimization systematically evaluated ring size, substituents, electronic effects, and stereochemistry to probe effects on loading, coupling, and overall stability with $\psi^{O}$ emerging as the only viable candidate. The synthesis outlined in Figure 2A requires three simple, scalable steps ( $>50 \mathrm{~g}$ ). In a similar fashion to $\psi^{2}$, inexpensive $\mathrm{P}_{2} \mathrm{~S}_{5}$ was reacted with 4-bromothiophenol yielding $\mathrm{P}(\mathrm{V})$ intermediate (7) which, when combined with hydrogenated cis-limonene oxide (8), yields the PS reagent (9), desulfurization with $\mathrm{SeO}_{2}$ yields $\psi^{\mathrm{O}}$.

With these new reagents in hand, a side-by-side comparison with state-of-the-art P(III) chemistry was conducted (Figure 2B). For the installation of the $\mathrm{PS}_{2}$ linkage, the three-step P(III) approach to access simple dimer (10) via P(III)-adduct (12) resulted in ca. 7\% of the PS impurity resulting from desulfurization during the deprotection event. In contrast, utilizing $\psi^{2}$, dimer (10) was cleanly accessed in two steps via P(V) adduct (11) in $>99 \%$ purity. In order to field-test and contrast the synthesis of mixed PO/PS backbones by the two platforms, PS dimer (13) was subjected to a standard phosphoramidite coupling with (18) to yield protected trimer (14) which, upon oxidation of $\mathrm{P}(\mathrm{III})$ to the requisite $\mathrm{P}(\mathrm{V})$, resulted in rapid desulfurization [see ratio of $(\mathbf{1 5}) /(\mathbf{1 6})$ over time]. On the other hand, the redox-neutral $\mathrm{P}(\mathrm{V})$ approach employing $\mathrm{P}(\mathrm{V})$ adduct (17) cleanly delivered the unprotected mixed PS/PO trimer (19) without any loss of sulfur.

The final challenge that $\mathrm{P}(\mathrm{V})$-based reagents face is the longstanding perception that their diminished coupling rates preclude them from being employed in traditional automated oligonucleotide synthesis regimes. With a full suite of reagents in hand based on $\mathrm{P}(\mathrm{V})$, their coupling performance was evaluated side-by-side with canonical $\mathrm{P}(\mathrm{III})$ chemistry through kinetics studies (Figure 2C). In addition, the original P(V)-based coupling using phosphotriester chemistry was included as this was the initial benchmark. Temporal reaction progress monitoring was performed by taking aliquots of the mixture coupling P-loaded-dT with AZT and monitoring product formation by HPLC/MS using an internal standard. Full kinetic profiles are included in the Supporting Information, and here we present the main trends by considering relative rates calculated from these profiles. Consistent with the literature, the classic P(V)-based phosphotriester approach was extremely sluggish, as revealed in the orange bar of Figure $2 \mathrm{C}$. However, as shown in Figure $2 \mathrm{C}$, the $\mathrm{P}(\mathrm{V})$-reagent suite detailed herein performed equally well to the industry-standard $\mathrm{P}(\mathrm{III})$ protocol with all reactions reaching full conversion in under two minutes.

Extensive optimization of the SPOS methodology for phosphoramidite-based synthesis has occurred over the course of the last $30+$ years. While some of these methods could be leveraged in this new context, there were areas where existing solutions were not compatible with the $P(V)$ synthesis protocol (Figure 3A). Notably, existing universal supports afforded insufficient stability toward DBU, prompting the development of a universal support (20) with significantly improved 
base stability. Guided by Stec's earlier work, Pya protecting groups were employed instead of the standard amide protecting groups (Figure 3B).). ${ }^{18}$ Improved results were also obtained when a Pom protection was employed for thymidine. ${ }^{35}$ With all $\mathrm{P}(\mathrm{V})$ reagents in hand and the chemoselectivity and relative coupling rates established, a systematic interrogation was undertaken to test the efficiency of this redox-neutral $\mathrm{P}(\mathrm{V})$ platform on automated solid-phase oligonucleotide synthesis (SPOS, Figure 3A). The cycle commences with the deblocking of the DMTr protecting group of a resin-bound nucleoside to afford a free 5 '-alcohol that is primed to react with any $\mathrm{P}(\mathrm{V})$-loaded nucleotide in the subsequent coupling step. This key step was carefully optimized for all reagents through systematic reactivity and hydrolysis studies using UV and ${ }^{31} \mathrm{P}$ NMR analyses to enable a robust double coupling protocol for each $\mathrm{P}(\mathrm{V})$ reagent. The subsequent capping and deblocking steps complete the solid-phase cycle and set the stage for the next coupling (Figure 3A). The utility of any new reagent system for an oligonucleotide synthesis platform is wedded to its fidelity and robustness in the context of preparing diverse sequences with a single protocol. Thus, a matrix was designed to incorporate all possible nucleobase (A, C, G, T) and sugar (DNA, LNA) combinations templated onto a 3-10-3 DNA/LNA gapmer scaffold, the current state of the art in RNase $\mathrm{H}$ activating ASOs (Figure 3C).). ${ }^{3}$ LNA modifications which have a dramatic effect on binding affinity were coupled in near quantitative yields. ${ }^{36}$ A single protocol was employed regardless of the $\mathrm{P}(\mathrm{V})$ monomers used (rather than tedious sequence-specific optimizations) - done to assess generality of the method versus sequence specific optimization. First, the general method was field-tested to produce homogeneous, chiral PS-ASOs with both alternating $(\mathbf{2 1}, \mathbf{2 2})$ and continuous stereochemical patterns (23-26)). This represents the second industrially viable platform to produce stereopure PS-ASOs, and the first to employ redox-neutral, sustainably derived $\mathrm{P}(\mathrm{V})$-based reagents. ${ }^{37}$ With this milestone achieved, the incorporation of $\mathrm{PO}_{2}$ linkages into these constructs was pursued. These chimeric sequences (27-30) could be accessed in high purity with no significant loss of sulfur during synthesis. Next, sequences bearing both PS and $\mathrm{PS}_{2}$ (31-34) linkages were prepared. Finally, constructs containing all four possible linkages were cleanly produced $(35,36)$. Thus, these protocols describe a convenient single platform for probing linkage SAR that could enable systematic tuning of physical and biological properties. ${ }^{38}$, ${ }^{39}$ Given the differences in scale, chemical sequence, method of purification and quantification, a direct comparison between yields of this $\mathrm{P}(\mathrm{V})$-platform and those of other stereopure methods is outside of the scope of this communication. In its present manifestation, these novel, homogeneous, chimeric oligonucleotides (21-38) were produced in $12-27 \%$ isolated yield using a sequence and linkage agnostic protocol. When compared directly to the stereorandom constructs (produced using state-of-the-art chemistry) that were produced in 30-60\% yield, and the ability to prepare unique chimeric systems, this represents a relatively small gap to fill to bring homogeneous, stereopure oligonucleotides on par with their stereorandom counterparts. From a pragmatic perspective the observed yields even in this first disclosure are more than enough to progress a medicinal chemistry program.

Current methods to produce PS oligonucleotides generate a statistical mixture of isomers depending on the specific conditions used. ${ }^{40}$ While homogeneous, well-defined ASOs are of value, no true $\mathrm{P}(\mathrm{V})$ oligonucleotide synthesis platform would be complete without rapid access to 


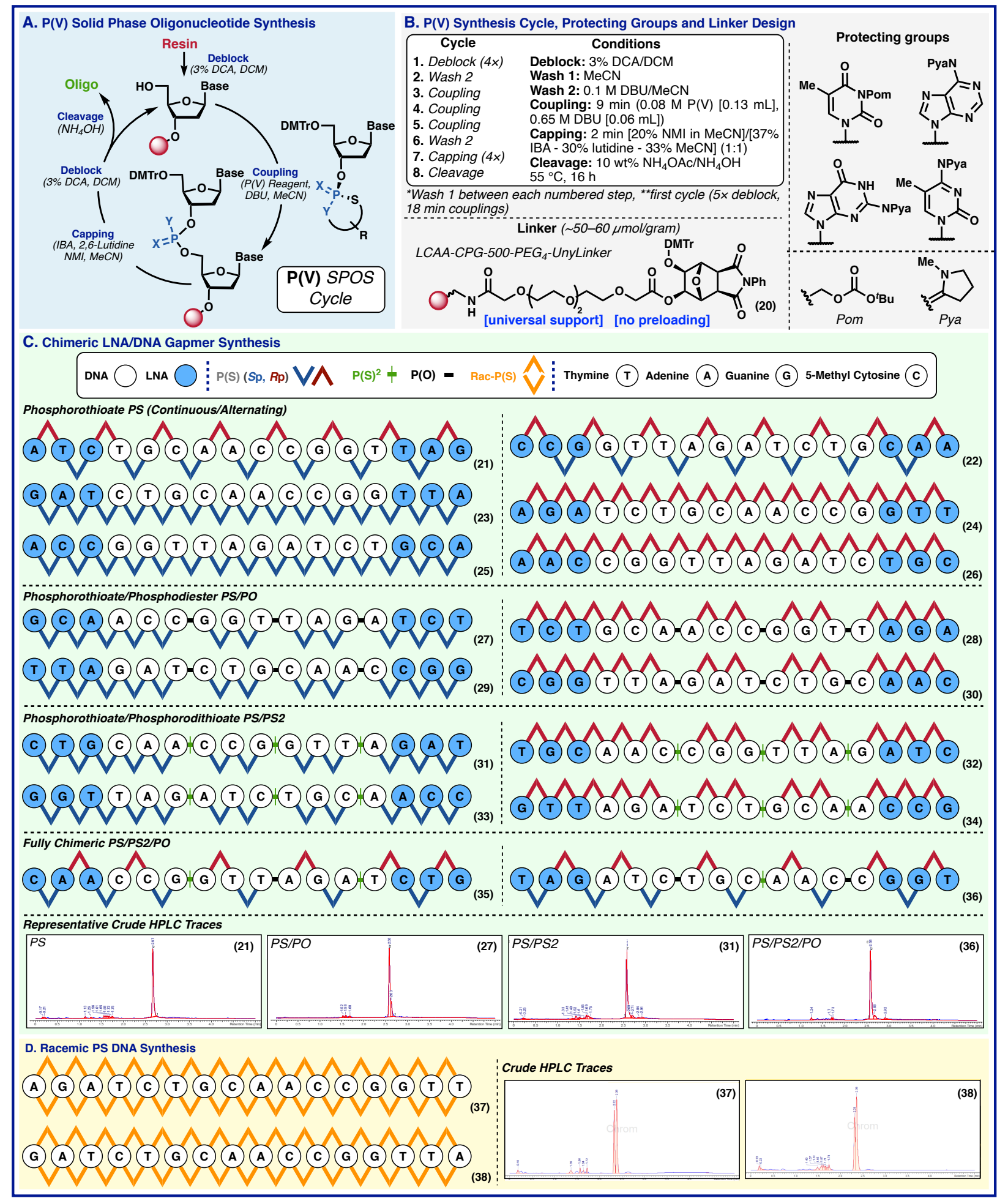

Fig. 3. Automated $P(V)$ oligonucleotide synthesis $(A) P(V)$ Solid-Phase oligonucleotide synthesis cycle (B) Synthetic cycle conditions, protecting groups and linker chemistry (C) 3-10-3 LNA/DNA 
gapmer synthesis (D) racemic phosphorothioate oligonucleotide synthesis. DCA, dichloroacetic acid; IBA, isobutryic anyhydride, NMI, N-methyl imidazole.

these stereorandom variants. ${ }^{41}$ Thus, a final need was to produce $r a c-\Psi$ (Figure 1), a derivative of $\Psi$ that retains high reactivity and provides mixtures of diastereomers comparable to those obtained with P(III) methodology. Rac- $\Psi$ was loaded onto DNA cores, and the corresponding monomers were cleanly implemented (Figure 3D) into the aforementioned workflow, thus enabling racemic PS oligos to be produced on this platform $(\mathbf{3 7}, \mathbf{3 8})$.

Oligonucleotide therapeutics, so-called informational drugs, target essentially every level of the central dogma. Given the immense chemical space conceivable, the number of nucleic acid modifications that have been investigated to date is quite narrow, with even fewer represented in the clinic. Although P(III)-based phosphoramidites have revolutionized access to vast swaths of this space, new linkages and chemical modifications have pushed the limits of what is currently accessible. Oligonucleotide synthesis originated with $\mathrm{P}(\mathrm{V})$-based reagents as Nature only uses this oxidation state to craft its building blocks of life, but it was cast away shortly thereafter due to perceptions of their reduced reactivity and selectivity. This work provides a compelling justification for renewed research in this field as it can enable democratized access to a wide range of medicinally promising chimeric sequences. Finally, this new approach offers a compelling opportunity to connect the exploration of new chemical space directly to patients through a platform offering improved environmental sustainability - enhancing our ability to both discover, develop and commercialize this promising class of therapeutics.

\section{ASSOCIATED CONTENT}

\section{Supporting Information}

Experimental procedures, characterizations, and the details of SPOS.

\section{AUTHOR INFORMATION}

\section{Corresponding Author}

*martin.eastgate@bms.com

*michael.schmidt@bms.com

*ivar.mcdonald@bms.com

*pbaran@scripps.edu

\section{Author Contributions}

The manuscript was written through contributions of all authors. All authors have given approval to the final version of the manuscript.

\section{ACKNOWLEDGMENT}

Financial support for this work was provided by Bristol Myers Squibb, NIGMS (GM106210), NIH (grant number GM-118176) and Marie Skłodowska-Curie Global Fellowships (749359- 
EnanSET, N.M.P) within the European Union research and innovation framework programme (2014-2020). We thank D.-H. Huang and L. Pasternack (Scripps Research) for assistance with NMR spectroscopy; J. Chen (Automated Synthesis Facility, Scripps Research) for purification of compounds and acquisition of HRMS data; A. L. Rheingold, C. E. Moore, and M. Gembicky (UCSD) for x-ray analysis and Subha Mukherjee (BMS) for calculating the PMI.

Competing interests. The authors declare no competing interests.

\section{References.}

1. Hughes, J.; Rees, S.; Kalindjian, S.; Philpott, K., Principles of early drug discovery. British Journal of Pharmacology 2011, 162 (6), 1239-1249.

2. Khvorova, A.; Watts, J. K., The chemical evolution of oligonucleotide therapies of clinical utility. Nat Biotechnol 2017, 35 (3), 238-248.

3. Wan, W. B.; Seth, P. P., The Medicinal Chemistry of Therapeutic Oligonucleotides. $J$ Med Chem 2016, 59 (21), 9645-9667.

4. Byrne, M.; Vathipadiekal, V.; Apponi, L.; Iwamoto, N.; Kandasamy, P.; Longo, K.; Liu, F.; Looby, R.; Norwood, L.; Shah, A.; Shelke, J. D.; Shivalila, C.; Yang, H.; Yin, Y.;

Guo, L.; Bowman, K.; Vargeese, C., Stereochemistry Enhances Potency, Efficacy, and Durability of Malat1 Antisense Oligonucleotides In Vitro and In Vivo in Multiple Species. Transl Vis Sci Technol 2021, 10 (1), 23.

5. Liu, Y.; Dodart, J. C.; Tran, H.; Berkovitch, S.; Braun, M.; Byrne, M.; Durbin, A. F.; Hu, X. S.; Iwamoto, N.; Jang, H. G.; Kandasamy, P.; Liu, F.; Longo, K.; Ruschel, J.; Shelke, J.; Yang, H.; Yin, Y.; Donner, A.; Zhong, Z.; Vargeese, C.; Brown, R. H., Jr., Variantselective stereopure oligonucleotides protect against pathologies associated with C9orf72-repeat expansion in preclinical models. Nat Commun 2021, 12 (1), 847.

6. Bleicher, K.; Duschmalé, J.; Duschmalé, M. B.; Hansen, H. F.; Funder, E.; Koch, T.; Li, M.; Schaeublin, A.; Shu, X.; Wu, Y. Gapmer Oligonucleotides Comprising A Phosphorodithioate Internucleoside Linkage. 2019.

7. Bethge, L.; Hauptmann, J.; Frauendorf, C.; Weingärtner, A. Nucleic Acids For Inhibiting Expression Of A Target Gene Comprising Phosphorodithioate Linkages. 2019.

8. Tredenick, T. Oligonucleotides: Opportunities, Pipeline and Challenges. https://www.pharmamanufacturing.com/articles/2016/oligonucleotides-opportunities-pipelineand-challenges/.

9. Eckstein, F.; Armstrong, V. W.; Sternbach, H., Stereochemistry of polymerization by DNA-dependent RNA-polymerase from Escherichia coli: an investigation with a diastereomeric ATP-analogue. Proc Natl Acad Sci U S A 1976, 73 (9), 2987-90.

10. Wilk, A.; Stec, W. J., Analysis of oligo(deoxynucleoside phosphorothioate)s and their diastereomeric composition. Nucleic Acids Res 1995, 23 (3), 530-4.

11. Andrews, B. I.; Antia, F. D.; Brueggemeier, S. B.; Diorazio, L. J.; Koenig, S. G.; Kopach, M. E.; Lee, H.; Olbrich, M.; Watson, A. L., Sustainability Challenges and Opportunities in Oligonucleotide Manufacturing. J Org Chem 2021, 86 (1), 49-61.

12. Mamoru Shimizu, T. Asymmetric Auxiliary Group. 2020.

13. Oka, N.; Yamamoto, M.; Sato, T.; Wada, T., Solid-phase synthesis of stereoregular oligodeoxyribonucleoside phosphorothioates using bicyclic oxazaphospholidine derivatives as monomer units. J Am Chem Soc 2008, 130 (47), 16031-7. 
14. Suchsland, R.; Appel, B.; Virta, P.; Muller, S., Synthesis of fully protected trinucleotide building blocks on a disulphide-linked soluble support. Rsc Advances 2021, 11 (7), 3892-3896.

15. Knouse, K. W.; deGruyter, J. N.; Schmidt, M. A.; Zheng, B.; Vantourout, J. C.; Kingston, C.; Mercer, S. E.; McDonald, I. M.; Olson, R. E.; Zhu, Y.; Hang, C.; Zhu, J.; Yuan, C.; Wang, Q.; Park, P.; Eastgate, M. D.; Baran, P. S., Unlocking P(V): Reagents for chiral phosphorothioate synthesis. Science 2018, 361 (6408), 1234-1238.

16. Stec, W. J.; Grajkowski, A.; Koziolkiewicz, M.; Uznanski, B., Novel route to oligo(deoxyribonucleoside phosphorothioates). Stereocontrolled synthesis of P-chiral oligo(deoxyribonucleoside phosphorothioates). Nucleic Acids Res 1991, 19 (21), 5883-8.

17. Stec, W. J.; Grajkowski, A.; Kobylanska, A.; Karwowski, B.; Koziolkiewicz, M.; Misiura, K.; Okruszek, A.; Wilk, A.; Guga, P.; Boczkowska, M., Diastereomers of Nucleoside 3'-O-(2-Thio-1,3,2-Oxathia(Selena)Phospholanes) - Building-Blocks for Stereocontrolled Synthesis of Oligo(Nucleoside Phosphorothioate)S. Journal of the American Chemical Society 1995, 117 (49), 12019-12029.

18. Okruszek, A.; Sierzchala, A.; Fearon, K. L.; Stec, W. J., Synthesis of Oligo(Deoxyribonucleoside Phosphorodithioate)S by the Dithiaphospholane Approach. Journal of Organic Chemistry 1995, 60 (21), 6998-7005.

19. Stec, W. J.; Karwowski, B.; Boczkowska, M.; Guga, P.; Koziołkiewicz, M.; Sochacki, M.; Wieczorek, M. W.; Błaszczyk, J., Deoxyribonucleoside 3'-O-(2-Thio- and 2-Oxo-“spiro"4,4-pentamethylene-1,3,2-oxathiaphospholane)s: Monomers for Stereocontrolled Synthesis of Oligo(deoxyribonucleoside phosphorothioate)s and Chimeric PS/PO Oligonucleotides§. Journal of the American Chemical Society 1998, 120 (29), 7156-7167.

20. Guga, P.; Stec, W. J., Synthesis of phosphorothioate oligonucleotides with stereodefined phosphorothioate linkages. Curr Protoc Nucleic Acid Chem 2003, Chapter 4 (1), Unit 417.

21. Eckstein, F.; Rizk, I., Synthesis of oligonucleotides by use of phosphoric triesters. Angew Chem Int Ed Engl 1967, 6 (8), 695-7.

22. Reese, C. B.; Saffhill, R., Oligonucleotide synthesis via phosphotriester intermediates: the phenyl-protecting group. Chemical Communications (London) 1968, (13), 767-768.

23. Letsinger, R. L.; Lunsford, W. B., Synthesis of thymidine oligonucleotides by phosphite triester intermediates. J Am Chem Soc 1976, 98 (12), 3655-61.

24. Reese, C. B.; Skone, P. A., The Protection of Thymine and Guanine Residues in Oligodeoxyribonucleotide Synthesis. Journal of the Chemical Society-Perkin Transactions 1 1984, (6), 1263-1271.

25. Wiesler, W. T.; Caruthers, M. H., Synthesis of Phosphorodithioate DNA via SulfurLinked, Base-Labile Protecting Groups1. The Journal of Organic Chemistry 1996, 61 (13), 4272-4281.

26. Yang, X., Solid-Phase Synthesis of RNA Analogs Containing Phosphorodithioate Linkages. Curr Protoc Nucleic Acid Chem 2017, 70 (1), 477 1-4 7713.

27. Guo, X.; Stolee, J. A.; Fillon, Y. A.; Zou, L. F., Trace-Level Determination of Acrylonitrile Generated in the Manufacturing Process of Oligonucleotides by Static Headspace Gas Chromatography with an Electron Impact(+) Mass Detector. Organic Process Research \& Development 2021, 25 (2), 318-326.

28. Capaldi, D. C.; Gans, H.; Krotz, A. H.; Arnold, J.; Carty, R. L.; Moore, M. N.;

Scozzari, A. N.; Lowery, K.; Cole, D. L.; Ravikumar, V. T., Synthesis of high-quality antisense 
drugs. Addition of acrylonitrile to phosphorothioate oligonucleotides: Adduct characterization and avoidance. Organic Process Research \& Development 2003, 7 (6), 832-838.

29. Oka, N.; Wada, T.; Saigo, K., An oxazaphospholidine approach for the stereocontrolled synthesis of oligonucleoside phosphorothioates. J Am Chem Soc 2003, 125 (27), 8307-17.

30. Porritt, G. M.; Reese, C. B., Use of the 2,4-dinitrobenzyl protecting group in the synthesis of phosphorodithioate analogues of oligodeoxyribonucleotides. Tetrahedron Letters 1990, 31 (9), 1319-1322.

31. Caruthers, M. H.; Beaton, G.; Cummins, L.; Dellinger, D.; Graff, D.; Ma, Y.-X.; Marshall, W. S.; Sasmor, H.; Shankland, P.; Van Wu, J.; Yau, E. K., Chemical and Biochemical Studies with Dithioate DNA. Nucleosides and Nucleotides 1991, 10 (1-3), 47-59. 32. Marshall, W. S.; Caruthers, M. H., Phosphorodithioate DNA as a potential therapeutic drug. Science 1993, 259 (5101), 1564-70.

33. Caruthers, M. H., The chemical synthesis of DNA/RNA: our gift to science. $J$ Biol Chem 2013, 288 (2), 1420-7.

34. Tabrizi, S. J.; Leavitt, B. R.; Landwehrmeyer, G. B.; Wild, E. J.; Saft, C.; Barker, R. A.; Blair, N. F.; Craufurd, D.; Priller, J.; Rickards, H.; Rosser, A.; Kordasiewicz, H. B.; Czech, C.; Swayze, E. E.; Norris, D. A.; Baumann, T.; Gerlach, I.; Schobel, S. A.; Paz, E.; Smith, A. V.; Bennett, C. F.; Lane, R. M.; Phase 1-2a, I.-H. S. S. T., Targeting Huntingtin Expression in Patients with Huntington's Disease. N Engl J Med 2019, 380 (24), 2307-2316. 35. de Koning, M. C.; Ghisaidoobe, A. B. T.; Duynstee, H. I.; Ten Kortenaar, P. B. W.; Filippov, D. V.; van der Marel, G. A., Simple and Efficient Solution-Phase Synthesis of Oligonucleotides Using Extractive Work-Up. Organic Process Research \& Development 2006, 10 (6), 1238-1245.

36. Singh, S. K.; Koshkin, A. A.; Wengel, J.; Nielsen, P., LNA (locked nucleic acids): synthesis and high-affinity nucleic acid recognition. Chemical Communications 1998, (4), 455456.

37. Iwamoto, N.; Butler, D. C. D.; Svrzikapa, N.; Mohapatra, S.; Zlatev, I.; Sah, D. W. Y.; Meena; Standley, S. M.; Lu, G.; Apponi, L. H.; Frank-Kamenetsky, M.; Zhang, J. J.; Vargeese, C.; Verdine, G. L., Control of phosphorothioate stereochemistry substantially increases the efficacy of antisense oligonucleotides. Nat Biotechnol 2017, 35 (9), 845-851.

38. Crooke, S. T.; Liang, X.-h.; Baker, B. F.; Crooke, R. M., Antisense Technology: A Review. Journal of Biological Chemistry.

39. Crooke, S. T.; Baker, B. F.; Crooke, R. M.; Liang, X. H., Antisense technology: an overview and prospectus. Nat Rev Drug Discov 2021.

40. Jahns, H.; Roos, M.; Imig, J.; Baumann, F.; Wang, Y.; Gilmour, R.; Hall, J., Stereochemical bias introduced during RNA synthesis modulates the activity of phosphorothioate siRNAs. Nat Commun 2015, 6 (1), 6317.

41. Ostergaard, M. E.; De Hoyos, C. L.; Wan, W. B.; Shen, W.; Low, A.; Berdeja, A.; Vasquez, G.; Murray, S.; Migawa, M. T.; Liang, X. H.; Swayze, E. E.; Crooke, S. T.; Seth, P. P., Understanding the effect of controlling phosphorothioate chirality in the DNA gap on the potency and safety of gapmer antisense oligonucleotides. Nucleic Acids Res 2020, 48 (4), 1691 1700. 